\title{
Caesarean section for deeply engaged foetal head.
}

\author{
Li Kwok To Thomas*
}

Department of Obstetrics and Gynecology, Queen Mary Hospital, Pokfulam, Hong Kong

Accepted on December 20, 2017

In Modern Medicine, Caesarean section for deeply engaged foetal head has increased in incidences worldwide. It is a currently a major challenge all over the world including but not limited to England and Hong Kong, China. If not handling properly this may lead to major mishaps and causing significant maternal and foetal morbidities such as asphyxia and beyond. However, the scientific evidence is scarce. This is partly due to low incidence previously and partly due to lack of awareness of this important condition.

Prevention is always better than cure used to be the Orthodox in Medicine and remains true in Modern Medicine. Prompt recognition of rapidly progressive labour is the first but important step to prevent this occurrence. Rapidly progressive labour may occur but not limit to placenta abruption, preterm labour too frequent uterine contraction with or without the use of Oxytocin. One of the sign will be abnormal foetal heart tracing such as variable deceleration.

If the indications of Caesarean section are abnormal continuous foetal heart monitoring, foetal distress, deep transverse arrest of labour, preterm labour or placental abruption, during the signing of consent and explanation to the patients should be provided about the possibility of rapidly progress of labour and reaching the second stage rapidly beforehand. Once confirmed, recourse to Instrumental delivery such as Low forceps delivery should be advised in the operating theatre. Prior to the Caesarean section, Foley catheter will be inserted. Vaginal examination should be performed in the same setting and these serve two purposes. The first one will be to check whether the cervix has been fully dilated and the level of the foetal head. If the cervix has been fully dilated and the foetal head is low down, the patient should be explained about this finding. Trial of instrumental delivery should be performed.

\section{Vaginal hand}

On the other hand, if the patient refused a trial of instrumental delivery or the cervix has not been fully dilated, the foetal head should be pushed back towards the head of the woman to facilitate Caesarean section.

\section{Assistant's vaginal hand to disimpact the foetal head}

Even during Caesarean section, if the Obstetrician found the foetal head is deeply engaged and the delivery is difficult. An assistant should be asked to disimpact the foetal head vaginally using a pair of sterile gloves after separating the patient's legs gently to explicit delivery.

\section{Platform}

The surgical bed should be at the lowest level during second stage Caesarean section. In addition, during the delivery of foetal head from the pelvis, the surgeon must be standing on a small platform to facility the delivery. This will prevent the shoulder joint of the surgeon being in flexion. In addition, the surgeon's elbow joint will be completely extended and is more effective physically in lifting out the head of the foetus.

\section{Locking of surgeon right elbow by his/her left hand}

The surgeon's right elbow should be extended and locked i.e., support by one's left hand in order to provide additional force to lift out the foetal head from the pelvis.

\section{Maternal head tilted down}

The surgical bed can be tiled in maternal head down position. In modern Medicine, spinal anaesthesia is the preferred mode of anaesthesia. Up to the level of T6 nerve will be adequate for Caesarean section. Before the surgeon would like to ask for maternal head tilted down position, it will always a good practice to discuss with the Anaesthetist and to avoid anaesthesia involving higher thoracic level thus causing respiratory compromise.

\section{Surgeon's hand beyond the foetal head}

During delivery, the foetal head should be disimpact before lifting out of the pelvis instead of lifting out directly from the pelvis in order to avoid lateral extension of the Kerr's incision. Lateral extension of the Kerr's incision may lead to torrential bleeding if involving bilateral uterine arteries. Furthermore, this may render the patient needs to repeat Caesarean section in future.

\section{Uterine muscle relaxant}

These include Ritodrine, Terbutaline and Nitroglycerin. They may help to relax the tight muscle in order to facilitate delivery. However, there is no evidence saying that one is more useful than the others and whether it is useful in this clinical condition is subject to debate.

\section{Patwardhan's method (shoulder first method)}

This method is said to be useful in occipital transverse and occipital anterior positions with the foetal head deeply impact in the pelvis. The uterine incision will be at the level of anterior shoulder. However, one must have pointed out that the most difficult delivery remains to be occipital posterior position but 
unfortunately this method cannot be applied. In fact, this may lead to extension of the Kerr's incision due to its large presenting diameter and the fact that flexion is necessary before the delivery of the foetal head. This translates into more manoeuvres and hence more complications may be encountered.

My approach will be a modified one from the above. Kerr's incision will be made as usual which is more familiar to most surgeons and may save time in identification of incision over the foetal shoulders as described by Patwardhan. If the incision is just over the foetal shoulders, the leading shoulder will be pushed toward the maternal head followed by the opposite shoulder. This manoeuvre will be carried on in stage until the surgeon's hand can be inserted beneath the foetal head to facilitate the delivery of the foetal head.

\section{The foetal disimpacting system and foetal pillow}

This is being one of the latest push methods. Around $180 \mathrm{~mL}$ normal saline will be filled into the pillow (in the form of balloon). It will be very interesting to know whether this is effective or not. From my point of view, this will be more useful for new surgeons.

\section{Reverse breech extraction}

After Kerr's incision, the surgeon's right hand will be introduced into the upper uterine cavity. Both of the foetal feet will be grasped and gently pulls the foetus up to facilitate delivery. Jeve in his meta-analysis had found significant lower maternal risks compared with the push method using a primary outcome measure of uterine incision extension [1]. However, it is importance to point out most surgeons had not been trained for such method of delivery. In their learning curve, more foetal injury may be encountered. Fracture of foetal humerus had been reported.
Emergency Lower Segment Caesarean section for deeply engaged foetal head is emerging. These may cause significant maternal and foetal morbidities. These includes but not limited to uterine tear, primary postpartum hemorrhage, low Apgar score, Perinatal depression, foetal injury including fracture humerus. These operations tend to be performed by more junior Obstetricians in an emergency condition [2]. Hence, they may not be able to recognize and to handle the possible complications. Teaching, sharing of experience, publication and training in the form of Obstetrics Drills will be useful to tackle this problem.

\section{References}

1. Jeve YB, Navti OB, Konje JC. Comparison of techniques used to deliver a deeply impacted fetal head at full dilation: A systematic review and meta-analysis. BJOG. 2016;123:337-45.

2. Chopra S, Bagga R, Keepanasseril A, et al. Disengagement of the deeply engaged fetal head during cesarean section in advanced labor: Conventional method versus reverse breech extraction. Acta Obstetricia Et Gynecologica. 2009;88:1163-6.

\section{*Correspondence to}

Dr. Li Kwok To Thomas

Department of Obstetrics and Gynecology

Queen Mary Hospital, Pokfulam

Hong Kong

E-mail: liktt@hku.hk 